\title{
Two Proofs and One Algorithm Related to the Analytic Hierarchy Process
}

\author{
Miron Pavluš, ${ }^{1}$ Rostislav Tomeš $\mathbb{D}^{\mathbb{D}},{ }^{2}$ and Lukáš Malec $\mathbb{D D}^{2}$ \\ ${ }^{1}$ Faculty of Management, University of Prešov, Konštantínova 16, 08001 Prešov, Slovakia \\ ${ }^{2}$ University of Business in Prague, Spálená 14, 11000 Prague, Czech Republic \\ Correspondence should be addressed to Rostislav Tomeš; tomes@vso-praha.eu
}

Received 30 April 2018; Revised 1 August 2018; Accepted 23 August 2018; Published 2 December 2018

Academic Editor: Wei-Chiang Hong

Copyright (C) 2018 Miron Pavluš et al. This is an open access article distributed under the Creative Commons Attribution License, which permits unrestricted use, distribution, and reproduction in any medium, provided the original work is properly cited.

\begin{abstract}
36 years ago, Thomas Saaty introduced a new mathematical methodology, called Analytic Hierarchy Process (AHP), regarding the decision-making processes. The methodology was widely applied by Saaty and by other authors in the different human activity areas, like planning, business, education, healthcare, etc. but, in general, in the area of management. In this paper, we provide two new proofs for well-known statement that the maximal eigenvalue $\lambda_{\max }$ is equal to $n$ for the eigenvector problem $A w=\lambda w$, where $A$ is, so-called, the consistent matrix of pairwise comparisons of type $n \times n(n \geq 2)$ with the solution vector $w$ that represents the probability components of disjoint events. Moreover, we suggest an algorithm for the determination of the eigenvalue problem solution $A w=$ $n w$ as well as the corresponding flowchart. The algorithm for arbitrary consistent matrix $A$ can be simply programmed and used.
\end{abstract}

\section{Introduction}

Literature regarding the Analytic Hierarchy Process is rather extensive. The well-known database Current Contents Connect provides a growing number of records in all document types (articles, books, reports, reviews, etc.) regarding the acronym AHP in a publication title. For the last 15 years, the number of records can be seen in Figure 1. These publications are mainly focused on different AHP applications, for example, a brown coal deposit [1], a comparative analysis of group aggregation techniques [2], a lab fire prevention management system [3], a green vendor evaluation and selection in production outsourcing in mining industry [4], an approximation of risk assessment [5], an evaluation of healthcare equipment [6], or many others.

Recently, the mathematical principles of AHP were published by Saaty [7]. This monograph involves a lot of mathematical findings that were collected in the area of decision-making processes, particularly, in the area of the AHP, starting from first publication [8] in 1980.

In this paper, we add two proofs regarding the main statement in AHP theory as well as a corresponding algorithm for practical use. Neither proofs nor algorithm has been involved in [7].
Let us consider a consistent matrix $A, n \times n(n \geq 2)$ in the form

$$
A=\left(\begin{array}{ccccc}
1 & a_{1} & a_{2} & \cdots & a_{n-1} \\
\frac{1}{a_{1}} & 1 & \frac{a_{2}}{a_{1}} & \cdots & \frac{a_{n-1}}{a_{1}} \\
\frac{1}{a_{2}} & \frac{a_{1}}{a_{2}} & 1 & \cdots & \frac{a_{n-1}}{a_{2}} \\
\vdots & \vdots & \vdots & \ddots & \vdots \\
\frac{1}{a_{n-1}} & \frac{a_{1}}{a_{n-1}} & \frac{a_{2}}{a_{n-1}} & \cdots & 1
\end{array}\right)
$$

where $a_{1}, a_{2}, \ldots, a_{n-1}$ are given positive constants, known in AHP theory as priorities. Let the column vector $w=$ $\left(p_{1}, p_{2}, \ldots p_{n}\right)^{\mathrm{T}}$ reflect the probabilities $p_{i}\left(p_{i} \geq 0\right)$ of disjoint events $\omega_{i}=1,2, \ldots, n$ so that the space $\Omega$ of elementary events $\omega_{i}$ is given as a union $\Omega=\omega_{1} \cup \omega_{2} \cup \ldots \omega_{n}$ of such events $\omega_{i}$, which are disjoint $\left(\omega_{i} \cap \omega_{j}=\oslash, i \neq j\right)$. Elementary events represent different criteria or alternatives in the AHP scheme 


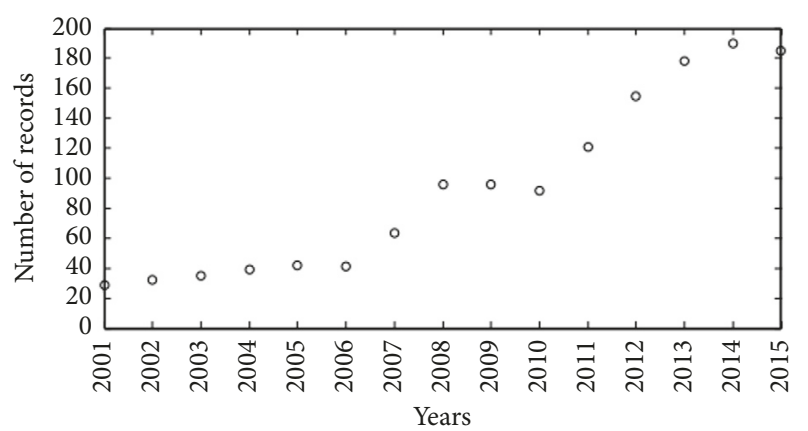

FIGURE 1: Records of AHP term in the database.

and probabilities $p_{i}=P\left(\omega_{i}\right)$ are known as the weights. As usual, it must be required

$$
\sum_{i=1}^{n} p_{i}=1
$$

for probability components $p_{i}$. Product $A w$ is a column vector whose components are sums of many addends if $n$ is sufficiently large natural number. Therefore, it is better to find a real number $\lambda$ such that the simpler product $\lambda w$ is equal to the complex product $A w$. Hence, we have the equation $A w=\lambda w$. Certainly, such equation has the trivial solution $w=\left(\begin{array}{llll}0 & 0 & \ldots\end{array}\right)^{\mathrm{T}}$ for arbitrary real number $\lambda$ but this is not interesting for us because this zero solution does not satisfy condition (2). If somebody is looking for nontrivial solutions $w \neq\left(\begin{array}{llll}0 & 0 & \ldots & 0\end{array}\right)^{\mathrm{T}}$, then the system $A w=\lambda w$, rewritten into the form $(A-\lambda E) w=\overrightarrow{0}$ must have a singular matrix $A-\lambda E$, i.e., determinant $|A-\lambda E|=0$. Here, $E$ marks the unit matrix.

\section{Derivation}

Now, we will demonstrate a way for how to derive the determinant $|A-\lambda E|$ for arbitrary consistent matrix $A$ of type $n \times n$. By the calculation of determinant $|A-\lambda E|$ [9-11], resp. by means of Laplace expansion the validity of following formula can be evaluated

$$
\begin{aligned}
|A-\lambda E| & =\left|\begin{array}{ccccc}
1-\lambda & a_{1} & a_{2} & \cdots & a_{n-1} \\
\frac{1}{a_{1}} & 1-\lambda & \frac{a_{2}}{a_{1}} & \cdots & \frac{a_{n-1}}{a_{1}} \\
\frac{1}{a_{2}} & \frac{a_{1}}{a_{2}} & 1-\lambda & \cdots & \frac{a_{n-1}}{a_{2}} \\
\vdots & \vdots & \vdots & \ddots & \vdots \\
\frac{1}{a_{n-1}} & \frac{a_{1}}{a_{n-1}} & \frac{a_{2}}{a_{n-1}} & \cdots & 1-\lambda
\end{array}\right| \\
& =(-\lambda)^{n-1}(n-\lambda) .
\end{aligned}
$$

The cases $n=2$ and $n=3$ are considered as special using fundamental rules like Sarrus. Next, we present two different ways for how to prove statement (3), which are involved neither in $[7,12]$ nor in other works known to the authors of this paper. The first proof is done by the direct calculation of the determinant on the left hand side of statement (3). The second proof is based on the mathematical induction method.

\section{Proof by Direct Calculation of Determinant}

At the beginning, we arrange the determinant of (3). We choose the factors $1 / a_{1}, 1 / a_{2}, \ldots, 1 / a_{n-1}$ from the second, third, fourth, up till $n$-th row. Thus, we get

$$
\begin{aligned}
& \left|\begin{array}{ccccc}
1-\lambda & a_{1} & a_{2} & \cdots & a_{n-1} \\
\frac{1}{a_{1}} & 1-\lambda & \frac{a_{2}}{a_{1}} & \cdots & \frac{a_{n-1}}{a_{1}} \\
\frac{1}{a_{2}} & \frac{a_{1}}{a_{2}} & 1-\lambda & \cdots & \frac{a_{n-1}}{a_{2}} \\
\vdots & \vdots & \vdots & \ddots & \vdots \\
\frac{1}{a_{n-1}} & \frac{a_{1}}{a_{n-1}} & \frac{a_{2}}{a_{n-1}} & \cdots & 1-\lambda
\end{array}\right| \\
& =\frac{1}{a_{1}} \frac{1}{a_{2}} \cdots \frac{1}{a_{n-1}} \\
& \times\left|\begin{array}{rrrrr}
1-\lambda & a_{1} & a_{2} & \cdots & a_{n-1} \\
1 & a_{1}(1-\lambda) & a_{2} & \cdots & a_{n-1} \\
1 & a_{1} & a_{2}(1-\lambda) & \cdots & a_{n-1} \\
\vdots & \vdots & \vdots & \ddots & \vdots \\
1 & a_{1} & a_{2} & \cdots & a_{n-1}(1-\lambda)
\end{array}\right| .
\end{aligned}
$$

Then, the factors $a_{1}, a_{2}, \ldots, a_{n-1}$ are set from the second, third, fourth, up till $n$-th column of the last determinant. We continuously obtain

$$
\frac{a_{1}}{a_{1}} \frac{a_{2}}{a_{2}} \cdots \frac{a_{n-1}}{a_{n-1}}\left|\begin{array}{ccccc}
1-\lambda & 1 & 1 & \cdots & 1 \\
1 & 1-\lambda & 1 & \cdots & 1 \\
1 & 1 & 1-\lambda & \cdots & 1 \\
\vdots & \vdots & \vdots & \ddots & \vdots \\
1 & 1 & 1 & \cdots & 1-\lambda
\end{array}\right| .
$$

By this way, we have arranged the determinant of (3) to the simple determinant

$$
\left|\begin{array}{ccccc}
z & 1 & 1 & \cdots & 1 \\
1 & z & 1 & \cdots & 1 \\
1 & 1 & z & \cdots & 1 \\
\vdots & \vdots & \vdots & \ddots & \vdots \\
1 & 1 & 1 & \cdots & z
\end{array}\right|
$$

of type $n \times n$, where $z=1-\lambda$. 
Next, we will express such determinant. By elimination in the first column and by selection of the common factor $(z-$ $1) / z$ from corresponding rows, we will get

$$
\left|\begin{array}{ccccc}
z & 1 & 1 & \cdots & 1 \\
1 & z & 1 & \cdots & 1 \\
1 & 1 & z & \cdots & 1 \\
\vdots & \vdots & \vdots & \ddots & \vdots \\
1 & 1 & 1 & \cdots & z
\end{array}\right|
$$$$
\left|\begin{array}{ccccc}
z & 1 & 1 & \cdots & 1 \\
0 & \frac{z^{2}-1}{z} & \frac{z-1}{z} & \cdots & \frac{z-1}{z} \\
0 & \frac{z-1}{z} & \frac{z^{2}-1}{z} & \cdots & \frac{z-1}{z} \\
\vdots & \vdots & \vdots & \ddots & \vdots \\
0 & \frac{z-1}{z} & \frac{z-1}{z} & \cdots & \frac{z^{2}-1}{z}
\end{array}\right|
$$$$
=\left(\frac{z-1}{z}\right)^{n-1}\left|\begin{array}{ccccc}
z & 1 & 1 & \cdots & 1 \\
0 & z+1 & 1 & \cdots & 1 \\
0 & 1 & z+1 & \cdots & 1 \\
\vdots & \vdots & \vdots & \ddots & \vdots \\
0 & 1 & 1 & \cdots & z+1
\end{array}\right| .
$$

The similar elimination in the second, third, fourth up till $n$ th column provides sequentially

$$
\begin{aligned}
& \left(\frac{z-1}{z}\right)^{n-1}\left|\begin{array}{ccccc}
z & 1 & 1 & \cdots & 1 \\
0 & z+1 & 1 & \cdots & 1 \\
0 & 1 & z+1 & \cdots & 1 \\
\vdots & \vdots & \vdots & \ddots & \vdots \\
0 & 1 & 1 & \cdots & z+1
\end{array}\right| \cdot \underset{\hookleftarrow}{\hookleftarrow} \\
& =\left(\frac{z-1}{z}\right)^{n-1}\left(\frac{z}{z+1}\right)^{n-2} \\
& \times\left|\begin{array}{ccccc}
z & 1 & 1 & \cdots & 1 \\
0 & z+1 & 1 & \cdots & 1 \\
0 & 0 & z+2 & \cdots & 1 \\
\vdots & \vdots & \vdots & \ddots & \vdots \\
0 & 0 & 1 & \cdots & z+2
\end{array}\right| \cdot-\frac{1}{z+2}=\ldots \\
& =\left(\frac{z-1}{z}\right)^{n-1}\left(\frac{z}{z+1}\right)^{n-2} \ldots\left(\frac{z+n-3}{z+n-2}\right)^{n-(n-1)}
\end{aligned}
$$

$$
\times\left|\begin{array}{ccccc}
z & 1 & 1 & \cdots & 1 \\
0 & z+1 & 1 & \cdots & 1 \\
0 & 0 & z+2 & \cdots & 1 \\
\vdots & \vdots & \vdots & \ddots & \vdots \\
0 & 0 & 0 & \cdots & z+n-1
\end{array}\right| .
$$

If we realize that the last determinant is equal to product of all diagonal elements and using elementary operations, the final result can be expressed

$$
(z-1)^{n-1}(z+n-1)
$$

which is the same result as the right hand side of statement (3), provided that $z=1-\lambda$.

\section{Proof by Mathematical Induction Method}

The equation (3) is valid for $n=2$. Next, we suppose that statement (3) holds for arbitrary natural $n(n \geq 2)$ and it will be proved that the statement is valid also for the next natural, i.e., $n+1$. So, we will prove that

$$
\left|\begin{array}{ccccc}
1-\lambda & a_{1} & a_{2} & \cdots & a_{n} \\
\frac{1}{a_{1}} & 1-\lambda & \frac{a_{2}}{a_{1}} & \cdots & \frac{a_{n}}{a_{1}} \\
\frac{1}{a_{2}} & \frac{a_{1}}{a_{2}} & 1-\lambda & \cdots & \frac{a_{n}}{a_{2}} \\
\vdots & \vdots & \vdots & \ddots & \vdots \\
\frac{1}{a_{n}} & \frac{a_{1}}{a_{n}} & \frac{a_{2}}{a_{n}} & \cdots & 1-\lambda
\end{array}\right|=(-\lambda)^{n}(n+1-\lambda) .
$$

By a similar way to that done in the beginning of Section 3, one can choose the factors $1 / a_{1}, 1 / a_{2}, \ldots, 1 / a_{n-1}$ from the second, third, fourth, up till $(n+1)$-th row and one can choose the factors $a_{1}, a_{2}, \ldots, a_{n}$ from the second, third, fourth, up till $(n+1)$-th column of the determinant in (10). Thus, we get the determinant

$$
\left|\begin{array}{ccccc}
z & 1 & 1 & \cdots & 1 \\
1 & z & 1 & \cdots & 1 \\
1 & 1 & z & \cdots & 1 \\
\vdots & \vdots & \vdots & \ddots & \vdots \\
1 & 1 & 1 & \cdots & z
\end{array}\right|
$$


of $n+1$ rows and $n+1$ columns. Next, we do the standard Laplace expansion, see, e.g. $[10,11]$ according to the last $(n+1)$ th row, so that

$$
\begin{aligned}
& \left|\begin{array}{ccccc}
z & 1 & 1 & \cdots & 1 \\
1 & z & 1 & \cdots & 1 \\
1 & 1 & z & \cdots & 1 \\
\vdots & \vdots & \vdots & \ddots & \vdots \\
1 & 1 & 1 & \cdots & z
\end{array}\right|_{(n+1) \times(n+1)} \\
& =(-1)^{n+1+1} 1\left|\begin{array}{ccccc}
1 & 1 & 1 & \cdots & 1 \\
z & 1 & 1 & \cdots & 1 \\
1 & z & 1 & \cdots & 1 \\
\vdots & \vdots & \vdots & \ddots & \vdots \\
1 & 1 & 1 & \cdots & 1
\end{array}\right|_{n \times n} \\
& +(-1)^{n+1+2} 1\left|\begin{array}{ccccc}
z & 1 & 1 & \cdots & 1 \\
1 & 1 & 1 & \cdots & 1 \\
1 & z & 1 & \cdots & 1 \\
\vdots & \vdots & \vdots & \ddots & \vdots \\
1 & 1 & 1 & \cdots & 1
\end{array}\right|_{n \times n}+\ldots \\
& +(-1)^{n+1+n+1} z\left|\begin{array}{ccccc}
z & 1 & 1 & \cdots & 1 \\
1 & z & 1 & \cdots & 1 \\
1 & 1 & z & \cdots & 1 \\
\vdots & \vdots & \vdots & \ddots & \vdots \\
1 & 1 & 1 & \cdots & z
\end{array}\right|_{n \times n} .
\end{aligned}
$$

Now, we arrange all determinants of type $n \times n$ so that the row of units is the first row. This can be achieved by switching adjacent rows around, which operation leads to a sign change of the determinant. Thus, we have

$$
\begin{aligned}
& \left|\begin{array}{ccccc}
z & 1 & 1 & \cdots & 1 \\
1 & z & 1 & \cdots & 1 \\
1 & 1 & z & \cdots & 1 \\
\vdots & \vdots & \vdots & \ddots & \vdots \\
1 & 1 & 1 & \cdots & z
\end{array}\right|_{(n+1) \times(n+1)} \\
& =(-1)^{n+1+1} 1\left|\begin{array}{ccccc}
1 & 1 & 1 & \cdots & 1 \\
z & 1 & 1 & \cdots & 1 \\
1 & z & 1 & \cdots & 1 \\
\vdots & \vdots & \vdots & \ddots & \vdots \\
1 & 1 & 1 & \cdots & 1
\end{array}\right|_{n \times n}
\end{aligned}
$$

$$
\begin{aligned}
& +(-1)^{n+1+2} 1(-1)\left|\begin{array}{ccccc}
1 & 1 & 1 & \cdots & 1 \\
z & 1 & 1 & \cdots & 1 \\
1 & z & 1 & \cdots & 1 \\
\vdots & \vdots & \vdots & \ddots & \vdots \\
1 & 1 & 1 & \cdots & 1
\end{array}\right|_{n \times n}+\ldots \\
& +(-1)^{n+1+n+1} z\left|\begin{array}{ccccc}
z & 1 & 1 & \cdots & 1 \\
1 & z & 1 & \cdots & 1 \\
1 & 1 & z & \cdots & 1 \\
\vdots & \vdots & \vdots & \ddots & \vdots \\
1 & 1 & 1 & \cdots & z
\end{array}\right|_{n \times n} .
\end{aligned}
$$

The multiple common determinant (with units in the first row) can be chosen after bracket and due to this, we obtain

$$
\begin{aligned}
& \sum_{i=1}^{n}(-1)^{n+1+i} 1(-1)^{i-1}\left|\begin{array}{ccccc}
1 & 1 & 1 & \cdots & 1 \\
z & 1 & 1 & \cdots & 1 \\
1 & z & 1 & \cdots & 1 \\
\vdots & \vdots & \vdots & \ddots & \vdots \\
1 & 1 & 1 & \cdots & 1
\end{array}\right|_{n \times n} \\
& +(-1)^{n+1+n+1} z\left|\begin{array}{ccccc}
z & 1 & 1 & \cdots & 1 \\
1 & z & 1 & \cdots & 1 \\
1 & 1 & z & \cdots & 1 \\
\vdots & \vdots & \vdots & \ddots & \vdots \\
1 & 1 & 1 & \cdots & z
\end{array}\right|_{n \times n}
\end{aligned}
$$

We arrange the sum near the first determinant in the form

$$
\sum_{i=1}^{n}(-1)^{n+1+i} 1(-1)^{i-1}=\sum_{i=1}^{n}(-1)^{n+2 i}=n(-1)^{n} .
$$

Then, the second determinant is substituted according to the induction assumption (3) which results in

$$
n(-1)^{n}\left|\begin{array}{ccccc}
1 & 1 & 1 & \cdots & 1 \\
z & 1 & 1 & \cdots & 1 \\
1 & z & 1 & \cdots & 1 \\
\vdots & \vdots & \vdots & \ddots & \vdots \\
1 & 1 & 1 & \cdots & 1
\end{array}\right|_{n \times n}+z(z-1)^{n-1}(z+n-1)
$$


We make the following arrangement of the last determinant. The first row of units from the rest of the rows is subtracted. Thus, the process continues into

$$
z(z-1)^{n-1}(z+n-1)+n(-1)^{n}\left|\begin{array}{ccccc}
1 & 1 & 1 & \cdots & 1 \\
z & 1 & 1 & \cdots & 1 \\
1 & z & 1 & \cdots & 1 \\
\vdots & \vdots & \vdots & \ddots & \vdots \\
1 & 1 & 1 & \cdots & 1
\end{array}\right|_{n \times n}
$$

Finally, the last determinantal operation is the expansion according to last column. Then, we get

$$
\begin{aligned}
& z(z-1)^{n-1}(z+n-1)+n(-1)^{n}(-1)^{n+1} \\
& \times\left|\begin{array}{ccccc}
z-1 & 0 & 0 & \cdots & 0 \\
0 & z-1 & 0 & \cdots & 0 \\
0 & 0 & z-1 & \cdots & 0 \\
\vdots & \vdots & \vdots & \ddots & \vdots \\
0 & 0 & 0 & \cdots & z-1
\end{array}\right|_{(n-1) \times(n-1)}
\end{aligned}
$$

or

$$
\begin{aligned}
z & (z-1)^{n-1}(z+n-1)+n(-1)^{n}(-1)^{n+1}(z-1)^{n-1} \\
& =(z-1)^{n-1}[z(z+n-1)-n] \\
& =(z-1)^{n-1}\left(z^{2}+z n-z-n\right) \\
& =(z-1)^{n-1}(z-1)(z+n)=(z-1)^{n}(z+n),
\end{aligned}
$$

provided $z=1-\lambda$. It is proven that the determinant on the left hand side of $(10)$ is equal to the expression on its right hand side. Thus, statement (3) holds for arbitrary natural $n(n \geq 2)$ as it results from mathematical induction methodology.

\section{Main Statement}

Both the proof by direct calculation of determinant in Section 3 and the proof by mathematical induction method in Section 4 lead to the same result; namely, the determinant $|A-\lambda E|$ is equal to $(-\lambda)^{n-1}(n-\lambda)$ for arbitrary consistent matrix $A$ with arbitrary natural $n(n \geq 2)$.

If somebody looks for the eigenvalues of the consistent matrix $A$ with arbitrary natural $n(n \geq 2)$, then the equation
$|A-\lambda E|=0$ holds, or $(-\lambda)^{n-1}(n-\lambda)=0$. From here, we have the following main theorem.

Theorem 1. The arbitrary consistent matrix $A$ with arbitrary natural $n(n \geq 2)$ has the following eigenvalues $\lambda_{1}=\lambda_{2}=$ $\ldots=\lambda_{n-1}=0$, but $\lambda_{n}=n$.

Hence, the maximal eigenvalue is $\lambda_{\max }=\lambda_{n}=n$. The reason why we are not interested in the other eigenvalues is simple: the components of eigenvectors $w=\left(p_{1} p_{2} \ldots p_{n}\right)^{\mathrm{T}}$ for the other eigenvalues do not fulfil the conditions $p_{i} \geq 0$, $i=1,2, \ldots, n$. We demonstrate this by the next simple example.

Example 1. Let us consider the following consistent matrix

$$
A=\left(\begin{array}{ccc}
1 & \frac{1}{3} & 2 \\
3 & 1 & 6 \\
\frac{1}{2} & \frac{1}{6} & 1
\end{array}\right)
$$

of type $3 \times 3$. According to Theorem 1 , the matrix has the following eigenvalues: $\lambda_{1}=\lambda_{2}=0$ and $\lambda_{3}=3$. Determine the eigenvectors of nonnegative components for eigenvalues $\lambda_{2}=0$ and $\lambda_{3}=3$.

Let $\lambda_{2}=0$. Then, the matrix $A-0 E$ equals

$$
\left(\begin{array}{ccc}
1 & \frac{1}{3} & 2 \\
3 & 1 & 6 \\
\frac{1}{2} & \frac{1}{6} & 1
\end{array}\right)
$$

and the system $(A-0 E) w=\overrightarrow{0}$ can be solved by the elimination method in the way

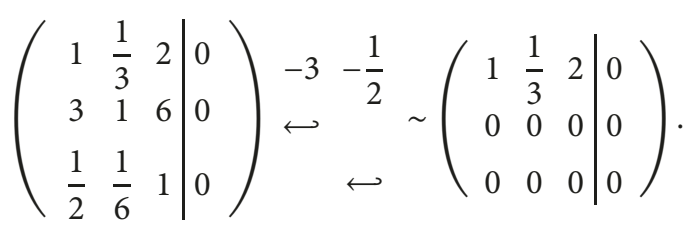

Hence, we have one equation $p_{1}+p_{2} / 3+2 p_{3}=0$ with three unknowns: $p_{1}, p_{2}$, and $p_{3}$. Moreover, the solution $w=$ $\left(\begin{array}{lll}p_{1} & p_{2} & p_{3}\end{array}\right)^{\mathrm{T}}$ must fulfil condition $(2)$ that means $p_{1}+p_{2}+p_{3}=$ 1. So, we solve two equations with three unknowns: $p_{1}, p_{2}$, and $p_{3}$. One unknown, let us say $p_{3}$, is a free one, and after substitution, one can get $p_{1}=-1 / 2-5 / 2 p_{3}$. This means if $p_{3} \geq 0$ then $p_{1}<0$ which is not feasible. 
On the other hand, $\lambda_{3}=3$. Then, the system $(A-3 E) w=$ $\overrightarrow{0}$ by the same elimination provides

$$
\begin{aligned}
& \left(\begin{array}{ccc|c|c|c}
-2 & \frac{1}{3} & 2 & 0 \\
3 & -2 & 6 & 0 & \frac{3}{2} & \frac{1}{4} \\
\frac{1}{2} & \frac{1}{6} & -2 & 0
\end{array}\right) \longleftrightarrow \\
& \sim\left(\begin{array}{ccc|c}
-2 & \frac{1}{3} & 2 & 0 \\
0 & -\frac{3}{2} & 9 & 0 \\
0 & \frac{1}{4} & -\frac{3}{2} & 0
\end{array}\right) \underset{⿱}{6} \\
& \sim\left(\begin{array}{ccc|c}
-2 & \frac{1}{3} & 2 & 0 \\
0 & -\frac{3}{2} & 9 & 0 \\
0 & 0 & 0 & 0
\end{array}\right)
\end{aligned}
$$

and including condition (2) one can get the unique solution $w=(2 / 92 / 31 / 9)^{\mathrm{T}}$, where all components are nonnegative. Thus, the result $\lambda=\lambda_{\max }$ is evident.

\section{Algorithm for Determination of Components}

In this section, we will demonstrate an algorithm which determines all components $p_{i} \geq 0, i=1,2, \ldots, n$, of the solution $w=\left(p_{1} p_{2} \ldots p_{n}\right)^{\mathrm{T}}$ if a consistent matrix $A$ of type $n \times n$ is given by arbitrary natural $n(n \geq 2)$; see (1). As we have seen in Section 4 , it must be solved by $(A-\lambda E) w=\overrightarrow{0}$ with $\lambda=$ $\lambda_{\max }=n$; otherwise some components $p_{i}$ can be negative and therefore these components do not represent probabilities of any event. In this case, the system $(A-n E) w=\overrightarrow{0}$ has the explicit form

$$
\begin{gathered}
\left(\begin{array}{ccccc}
1-n & a_{1} & a_{2} & \cdots & a_{n-1} \\
\frac{1}{a_{1}} & 1-n & \frac{a_{2}}{a_{1}} & \cdots & \frac{a_{n-1}}{a_{1}} \\
\frac{1}{a_{2}} & \frac{a_{1}}{a_{2}} & 1-n & \cdots & \frac{a_{n-1}}{a_{2}} \\
\vdots & \vdots & \vdots & \ddots & \vdots \\
\frac{1}{a_{n-1}} & \frac{a_{1}}{a_{n-1}} & \frac{a_{2}}{a_{n-1}} & \cdots & 1-n
\end{array}\right)\left(\begin{array}{c}
p_{1} \\
p_{2} \\
p_{3} \\
\vdots \\
p_{n}
\end{array}\right) \\
=\left(\begin{array}{c}
0 \\
0 \\
0 \\
\vdots \\
0
\end{array}\right)
\end{gathered}
$$

which is arranged by the following way. First, we multiply the second, third, up to $n$-th row by $a_{1}, a_{2}, \ldots, a_{n-1}$. The following is obtained

$$
\begin{aligned}
& \left(\begin{array}{ccccc}
1-n & a_{1} & a_{2} & \cdots & a_{n-1} \\
1 & a_{1}(1-n) & a_{2} & \cdots & a_{n-1} \\
1 & a_{1} & a_{2}(1-n) & \cdots & a_{n-1} \\
\vdots & \vdots & \vdots & \ddots & \vdots \\
1 & a_{1} & a_{2} & \cdots & a_{n-1}(1-n)
\end{array}\right)\left(\begin{array}{c}
p_{1} \\
p_{2} \\
p_{3} \\
\vdots \\
p_{n}
\end{array}\right) \\
& =\left(\begin{array}{c}
0 \\
0 \\
0 \\
\vdots \\
0
\end{array}\right) .
\end{aligned}
$$

Second, we rewrite the last system in the form

$$
\begin{aligned}
& \left(\begin{array}{ccccc}
1-n & 1 & 1 & \cdots & 1 \\
1 & 1-n & 1 & \cdots & 1 \\
1 & 1 & 1-n & \cdots & 1 \\
\vdots & \vdots & \vdots & \ddots & \vdots \\
1 & 1 & 1 & \cdots & 1-n
\end{array}\right)\left(\begin{array}{c}
p_{1} \\
a_{1} p_{2} \\
a_{2} p_{3} \\
\vdots \\
a_{n-1} p_{n}
\end{array}\right) \\
& =\left(\begin{array}{c}
0 \\
0 \\
0 \\
\vdots \\
0
\end{array}\right)
\end{aligned}
$$

to get a simpler matrix. Then, we use the elimination method as

$$
\left(\begin{array}{ccccc|c}
1-n & 1 & 1 & \cdots & 1 & 0 \\
1 & 1-n & 1 & \cdots & 1 & 0 \\
1 & 1 & 1-n & \cdots & 1 & 0 \\
\vdots & \vdots & \vdots & \ddots & \vdots & \vdots \\
1 & 1 & 1 & \cdots & 1-n & 0
\end{array}\right) . \begin{gathered}
-\frac{1}{1-n} \\
\hookleftarrow \\
\vdots
\end{gathered}
$$

$$
\begin{aligned}
& \sim\left(\begin{array}{ccccc|c}
1-n & 1 & 1 & \cdots & 1 & 0 \\
0 & 2-n & 1 & \cdots & 1 & 0 \\
0 & 1 & 2-n & \cdots & 1 & 0 \\
\vdots & \vdots & \vdots & \ddots & \vdots & \vdots \\
0 & 1 & 1 & \cdots & 2-n & 0
\end{array}\right) \cdot-\frac{1}{2-n} \\
& \sim \ldots \sim\left(\begin{array}{ccccc|c}
1-n & 1 & 1 & \cdots & 1 & 0 \\
0 & 2-n & 1 & \cdots & 1 & 0 \\
0 & 0 & 3-n & \cdots & 1 & 0 \\
\vdots & \vdots & \vdots & \ddots & \vdots & \vdots \\
0 & 0 & 0 & \cdots & 0 & 0
\end{array}\right) .
\end{aligned}
$$

We can see that the last row is zero which is evident due to determinant $|A-\lambda E|$ with the eigenvalue $\lambda=n$ being 
equal to zero. Thus, there exist many eigenvectors $w=$ $\left(p_{1} p_{2} \ldots p_{n}\right)^{\mathrm{T}}$ that satisfy (24). In this set, we choose only one eigenvector satisfying condition (2). This can be achieved by means of the substitution of zero row in the last system arrangement with the following row. Condition (2) can be written in the matrix form

$$
\left(\begin{array}{lllll}
1 & 1 & 1 & \cdots & 1
\end{array}\right)\left(\begin{array}{c}
p_{1} \\
p_{2} \\
p_{3} \\
\vdots \\
p_{n}
\end{array}\right)=(1) ;
$$

however, our consideration is related to the unknown

$$
\left(\begin{array}{c}
p_{1} \\
a_{1} p_{2} \\
a_{2} p_{3} \\
\vdots \\
a_{n-1} p_{n}
\end{array}\right)
$$

in the last system arrangement. So, condition (2) must be rewritten as

$$
\left(\begin{array}{lllll}
1 & \frac{1}{a_{1}} & \frac{1}{a_{2}} & \cdots & \frac{1}{a_{n-1}}
\end{array}\right)\left(\begin{array}{c}
p_{1} \\
a_{1} p_{2} \\
a_{2} p_{3} \\
\vdots \\
a_{n-1} p_{n}
\end{array}\right)=(1)
$$

and due to this, we will have the system

$$
\left(\begin{array}{ccccc|c}
1-n & 1 & 1 & \cdots & 1 & 0 \\
0 & 2-n & 1 & \cdots & 1 & 0 \\
0 & 0 & 3-n & \cdots & 1 & 0 \\
\vdots & \vdots & \vdots & \ddots & \vdots & \vdots \\
1 & \frac{1}{a_{1}} & \frac{1}{a_{2}} & \cdots & \frac{1}{a_{n-1}} & 1
\end{array}\right)
$$

for unknown vector (29).

A core of the suggested algorithm consists in the elimination of the last row of the system (31). This elimination can be processed by the following steps.

Step 1 . The first row in (31) is multiplied by fraction $-1 /(1-n)$ and the multiplied row is added to the last row. We denote the fraction by $c_{n-1}$. Due to this operation, the last modified row has the first element zero and the second element is $1 / a_{1}+c_{n-1}$.

Step 2. The second row in (31) is multiplied by fraction $-\left(1 / a_{1}+c_{n-1}\right) /(2-n)$ and the multiplied row is added to the last modified row. We denote the fraction by $c_{n-2}$. Due to this operation, the new modified row has the $r$-st two elements as zeros and the third element is $1 / a_{2}+c_{n-1}+c_{n-2}$.

Etc.

Step $n-1$. The $(n-1)$-st row in (31) is multiplied by fraction $-\left(1 / a_{n-2}+c_{n-1}+c_{n-2}+\ldots+c_{2}\right) /(-1)$ and the multiplied row is added to the last modified row. We denote the fraction by $c_{1}$. Due to such operation, the new modified row has the first $(n-1)$ elements as zeros and the last element is $1 / a_{n-1}+c_{n-1}+$ $c_{n-2}+\ldots+c_{1}$. A key point of elimination process is to get system (31) into the form

$$
\left(\begin{array}{ccccc|c}
1-n & 1 & 1 & \cdots & 1 & 0 \\
0 & 2-n & 1 & \cdots & 1 & 0 \\
0 & 0 & 3-n & \cdots & 1 & 0 \\
\vdots & \vdots & \vdots & \ddots & \vdots & 0 \\
0 & 0 & 0 & \cdots & c_{0} & 1
\end{array}\right),
$$

where

$$
c_{0}=\frac{1}{a_{n-1}}+c_{n-1}+c_{n-2}+\ldots+c_{1},
$$

as it follows from the elimination process. Thus, we come to Algorithm 1.

For programming purposes, however, the flowchart in Figure 2 is more suitable than the algorithm individual steps.

However, there are more ways for how to calculate the eigenvector components. In Sections 2 and 3 of this paper, we provided two proofs for well-known statement that $\lambda_{\text {max }}$ is equal to $n$ for the eigenvector problem $A w=\lambda w$. Thus, the system $(A-\lambda E) w=\overrightarrow{0}$ can be directly solved with $\lambda=\lambda_{\max }=n,(A-n E) w=\overrightarrow{0}$, where the components $p_{i}$ of vector $w=\left(p_{1}, p_{2}, \ldots p_{n}\right)^{\mathrm{T}}$ represent probabilities of any event. There is no need to calculate $\lambda_{\max }$ because $\lambda_{\max }=$ $n$. Algorithm 1 helps to calculate components $p_{1}, p_{2}, \ldots p_{n}$ directly using positive constants $a_{1}, a_{2}, \ldots, a_{n-1}$ from the first row of consistent pairwise matrix $A, n \times n(n \geq 2)$.

Example 2. We have the following consistent matrix

$$
A=\left(\begin{array}{cccccc}
1 & 2.414 & 3.166 & 4.138 & 4.138 & 5.379 \\
0.414 & 1 & 1.311 & 1.714 & 1.714 & 2.229 \\
0.316 & 0.763 & 1 & 1.307 & 1.307 & 1.699 \\
0.242 & 0.583 & 0.765 & 1 & 1.000 & 1.300 \\
0.242 & 0.583 & 0.765 & 1.000 & 1 & 1.300 \\
0.186 & 0.449 & 0.588 & 0.769 & 0.769 & 1
\end{array}\right)
$$

of type $6 \times 6$, which is pairwise matrix of quantitative criterion. We need to solve the system $(A-n E) w=$ $\overrightarrow{0}$. In this example, we exceptionally use, instead of standard mathematical tools, modeling in MS Excel, specifically using its additional tool Solver. We obtain vector $w=$

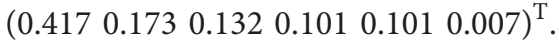


Input: $a_{1}, a_{2}, \ldots, a_{n-1}(n \geq 2)$

Output: $p_{1}, p_{2}, \ldots, p_{n}$

(1) $c_{n-1} \longleftarrow 1 /(n-1)$;

(2) while $n>2$ do

(3) for each $k \in\{2,3, \ldots, n-1\}$ do

(4) $c_{n-k} \longleftarrow\left(1 / a_{k-1}+\sum_{i=1}^{n-1} c_{n-i}\right) /(n-k)$;

(5) end

(6) end

(7) $c_{0} \longleftarrow 1 / a_{n-1}+\sum_{i=1}^{n-1} c_{n-i}$;

(8) $p_{n} \longleftarrow 1 /\left(c_{0} a_{n-1}\right)$;

(9) for each $k \in\{n-1, n-2, \ldots, 1\}$ do

(10) $p_{k} \longleftarrow 1 /\left[(n-k) a_{k-1}\right] \sum_{i=k+1}^{n} a_{i-1} p_{i}$;

(11) end $\longrightarrow$ given priorities

$\longrightarrow$ unknown eigenvector components

$\longrightarrow$ initial assignment

$\longrightarrow$ if $n=2$ then jump to step (7)

$\longrightarrow$ cycle for index $k$

$\longrightarrow c_{n-2}, c_{n-1}, \ldots, c_{1}$

$\longrightarrow$ relation (33)

$\longrightarrow$ last unknown component $p_{n}$

$\longrightarrow$ cycle for index $k$

$\longrightarrow p_{n-1}, p_{n-2}, \ldots, p_{1}$

Algorithm 1: Calculation of eigenvector components.

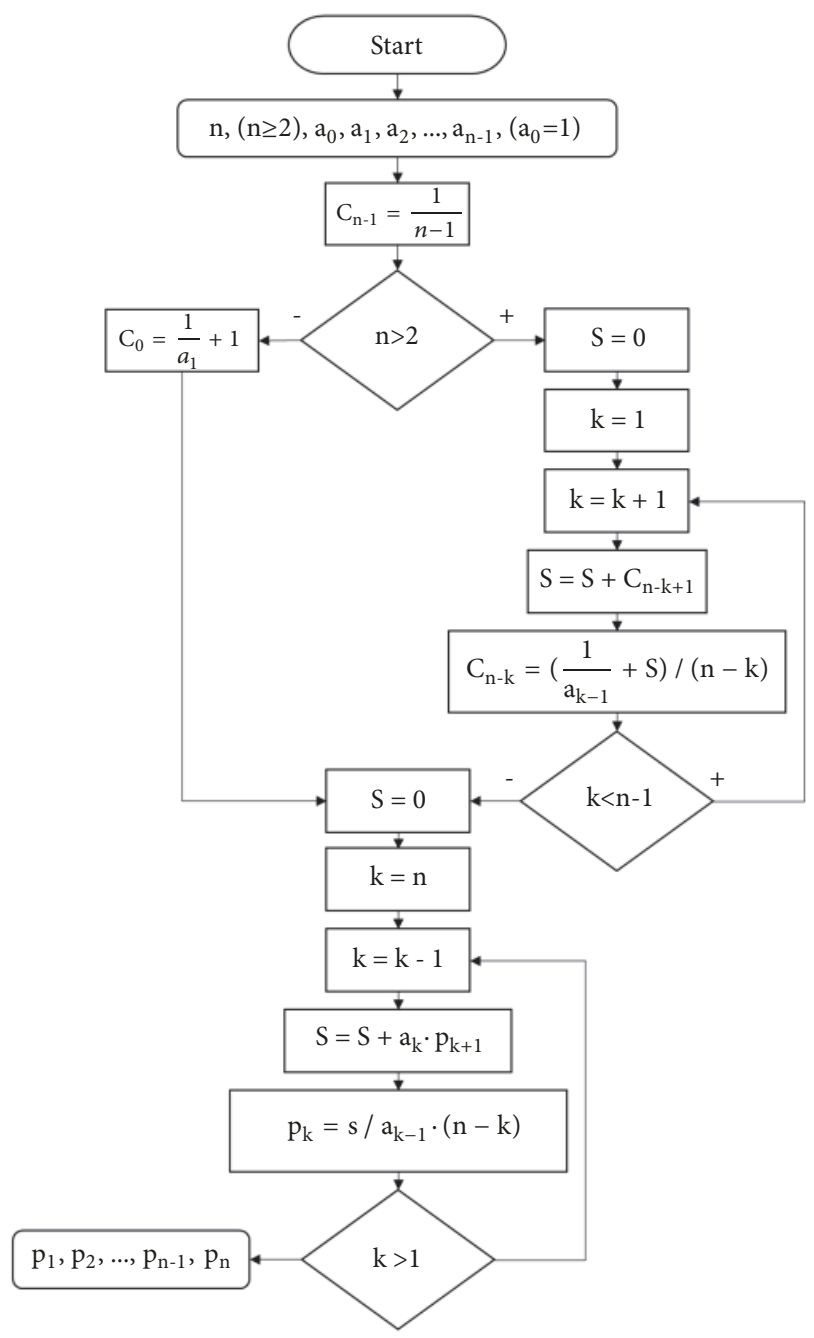

FIGURE 2: Flowchart of the algorithm

The constants $a_{1}=2.414, a_{2}=3.166, a_{3}=$ 4.138, $a_{4}=4.138$, and $a_{5}=5.379$ are used in the algorithm described in Section 6. The result is $w=$ $\left(\begin{array}{llllll}0.417 & 0.173 & 0.132 & 0.101 & 0.101 & 0.007\end{array}\right)^{\mathrm{T}}$. We can see that using such algorithm it gives the same results.

\section{Conclusion}

This paper has a mathematical methodological character utilizing only basic matrix theory. It shows two ways for how to prove the well-known statement regarding the set 
of eigenvalues of $A w=\lambda w$ with a consistent matrix $A$ of type $n \times n(n \geq 2)$, and it demonstrates how to find the corresponding eigenvector components presented as some probabilities of events for maximal eigenvalue $\lambda=\lambda_{\max }=n$.

The derived and suggested algorithm can be easily programmed in different languages $(\mathrm{C}++, \mathrm{C}, \mathrm{FORTRAN}$, MATHEMATICA, etc.) and can be used in AHP methodology for determination of weights (probabilities) when comparing the criteria to a goal or when comparing the alternatives to an individual criterion. During such comparison, it is important to make sure that the criteria or alternatives present a set of disjoint events $\left(\omega_{i} \cap \omega_{j}=\varnothing, i \neq j\right)$. If some events, say $\omega_{i}$ and $\omega_{j}$, are such that $\omega_{i} \cap \omega_{j}=\varnothing, i \neq j$, then condition (2) does not hold and the calculated weights do not reflect any real examined problem. In such a case, we suggest considering the intersection $\omega_{i} \cap \omega_{j}$ as a new event, say $\omega_{k}=\omega_{i} \cap \omega_{j}$, and incorporating this new event into the previous set of events. Next, we note that the statement and algorithm suggested in the paper hold for arbitrary natural $n(n \geq 2)$. This enables formulating and solving the relatively complex AHP problems with large number of criteria and huge number of alternatives like those in [1-6].

During practical evaluation of AHP, it can happen that the matrix $A$ is not consistent but is close to a consistent matrix $A^{\prime}$. In this case, certainly, $\lambda_{\max }(A)$ differs from $\lambda_{\max }\left(A^{\prime}\right)$ and the corresponding difference is measured by means of, socalled, consistency index

$$
\frac{\lambda(A)-n}{n-1}
$$

that was introduced by Saaty in [8]. If the consistency index is close to zero, then the suggested algorithm can be applied, but condition (2) holds only approximately. The usual problem of how to obtain $\lambda_{\max }(A)$ for a nonconsistent general matrix $A$ can be solved by means of the software packages which provide eigenvalues for the general full matrices. Such access, however, does not suppose any special matrix structure, like AHP matrix (1), and the eigenvalue software calculations in this general case are computationally difficult, especially when $n$ is large natural number. The special structure nonconsistent matrices which are close to AHP matrix are not considered in this paper and can be analyzed in the future research.

It can be said that there are some methods to repair consistency of matrices. Xu [13] defined a criterion to find unusual and false element and proposed method based on finding such elements in matrix and repair it. The repaired matrix has (after calculating new element instead of unusual one) an acceptable consistency. Saaty [12] proposed method based on additive perturbation using $a_{i j} w_{j} / w_{i}, i \neq j$, where $w$ is an eigenvector, and focused their attention on element of the matrix $A$ which provokes inconsistency. The consistency is generally more acceptable by substituting this element.

\section{Acknowledgments}

The KEGA-037PU-4/2014 and Grant Agency of Academic Alliance GA/13/2018 research supports are gratefully acknowledged.

\section{References}

[1] J. Blachowski, "Methodology for assessment of the accessibility of a brown coal deposit with Analytical Hierarchy Process and Weighted Linear Combination," Environmental Earth Sciences, vol. 74, no. 5, pp. 4119-4131, 2015.

[2] W. Ossadnik, S. Schinke, and R. H. Kaspar, "Group Aggregation Techniques for Analytic Hierarchy Process and Analytic Network Process: A Comparative Analysis," Group Decision and Negotiation, vol. 25, no. 2, pp. 421-457, 2016.

[3] C.-C. Shih, R. S. Horng, and S.-K. Lee, "Investigation of Lab Fire Prevention Management System of Combining Root Cause Analysis and Analytic Hierarchy Process with Event Tree Analysis," Mathematical Problems in Engineering, vol. 2016, Article ID 3161823, 12 pages, 2016.

[4] R. Sivakumar, D. Kannan, and P. Murugesan, "Green vendor evaluation and selection using AHP and Taguchi loss functions in production outsourcing in mining industry," Resources Policy, vol. 46, pp. 64-75, 2015.

[5] A. Kokangül, U. Polat, and C. Dağsuyu, "A new approximation for risk assessment using the AHP and Fine Kinney methodologies," Safety Science, vol. 91, pp. 24-32, 2017.

[6] S. Peregrin and J. Jablonsky, "Analytic hierarchy process as a tool for group evaluation of healthcare equipment," International Journal of Business and Systems Research, vol. 10, no. 2-4, pp. 124-141, 2016.

[7] T. L. Saaty, Mathematical Principles of Decision Making (Principia Mathematica Decernendi), RWS Publications, Pittsburg, Calif, USA, 2010.

[8] T. L. Saaty, The Analytic Hierarchy Process, McGraw-Hill, New York, NY, USA, 1980.

[9] A. J. Hobson, Just the MATHs, Mathcentre, Coventry, UK, 2002.

[10] R. Vein and P. Dale, Determinants and Their Application in Mathematical Physics, Springer, New York, NY, USA, 1999.

[11] J. Seman, E. Litavcová, and M. Pavluš, Mathematics for Managers, Bookman, Prešov, Slovakia, 2nd edition, 2012.

[12] T. L. Saaty, "Decision-making with the AHP: why is the principal eigenvector necessary," European Journal of Operational Research, vol. 145, no. 1, pp. 85-91, 2003.

[13] Y. Xu and H. Wang, "Eigenvector method, consistency test and inconsistency repairing for an incomplete fuzzy preference relation," Applied Mathematical Modelling, vol. 37, no. 7, pp. 5171-5183, 2013.

\section{Conflicts of Interest}

The authors declare that they have no conflicts of interest. 


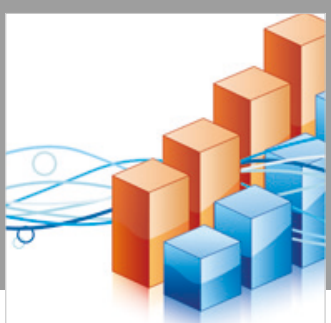

Advances in

Operations Research

\section{-n-m}
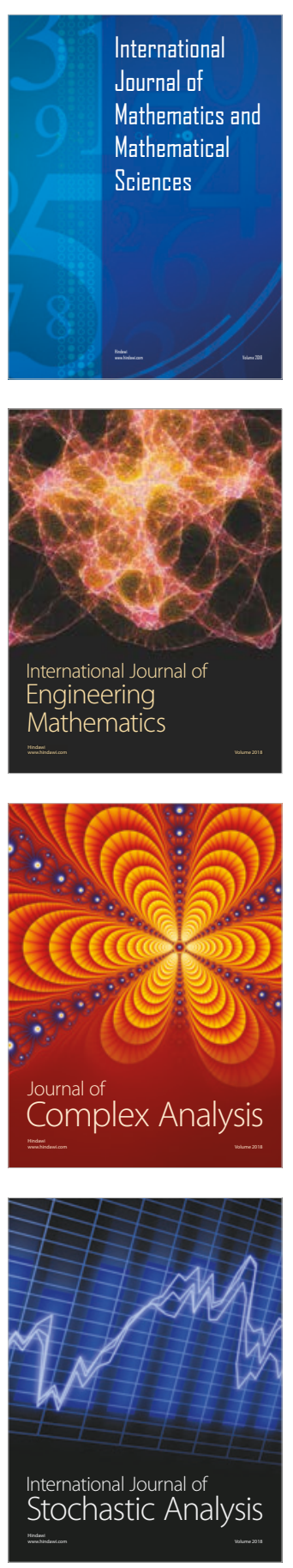
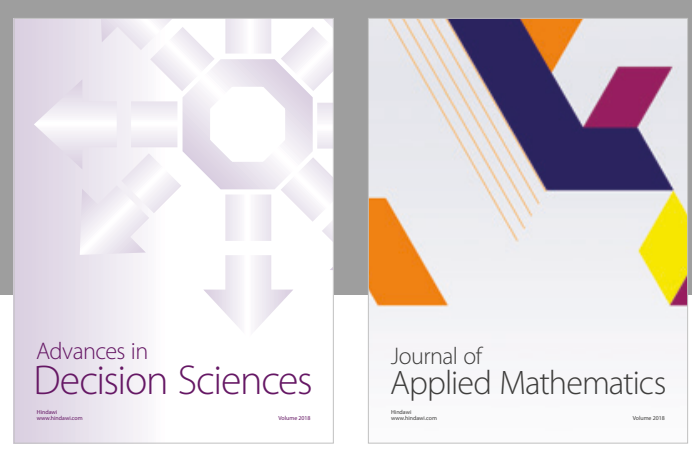

Journal of

Applied Mathematics
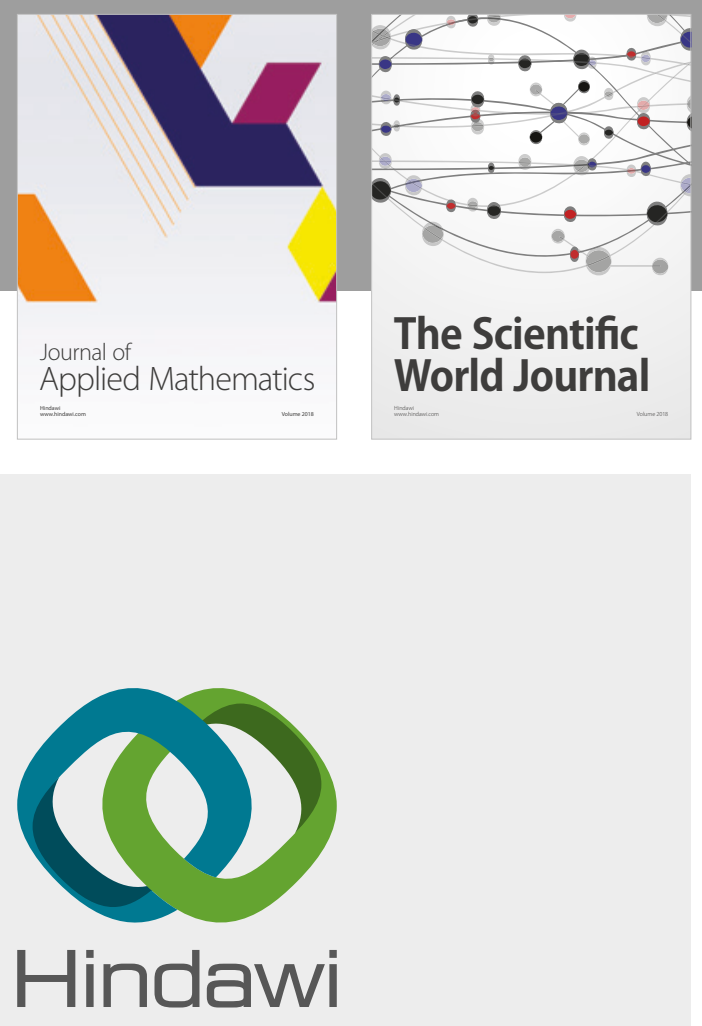

Submit your manuscripts at

www.hindawi.com

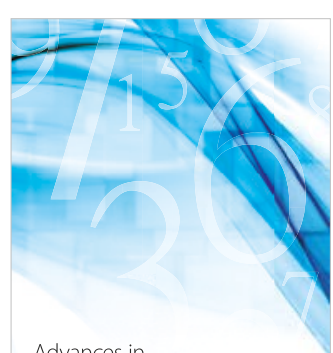

Advances in
Numerical Analysis
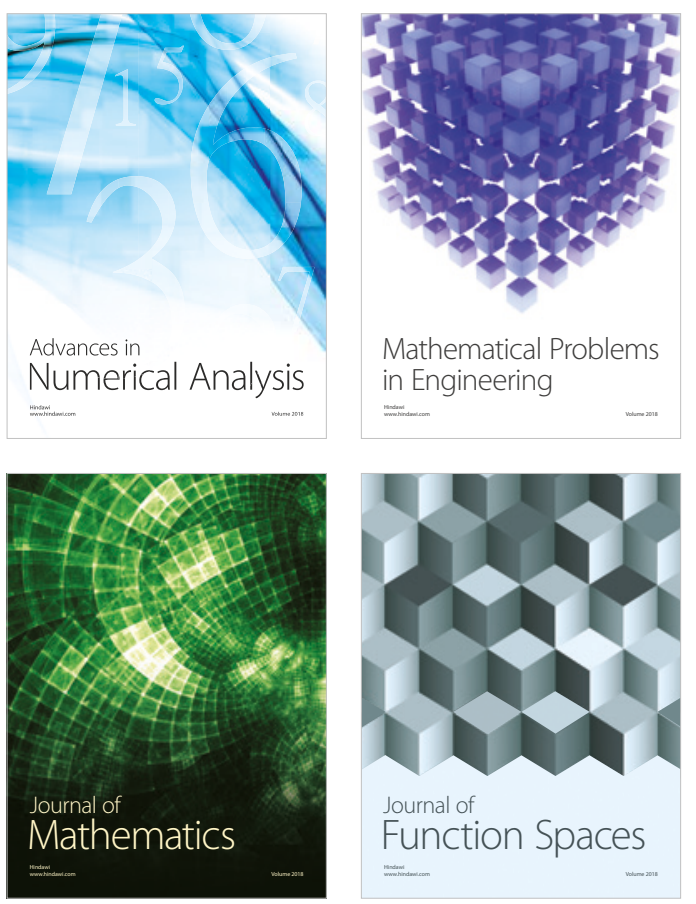

Mathematical Problems in Engineering

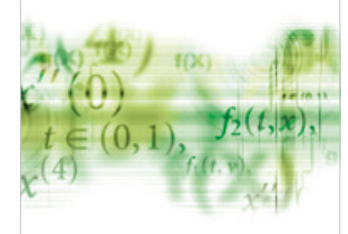

International Journal of

Differential Equations

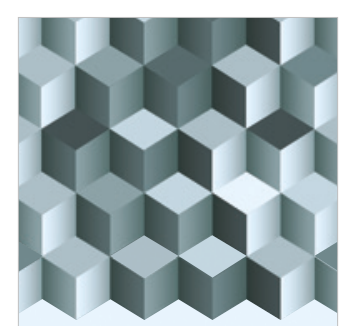

Journal of

Function Spaces

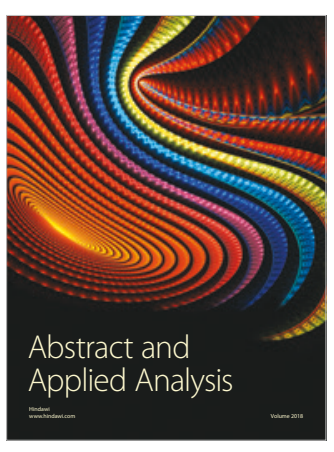

The Scientific

World Journal

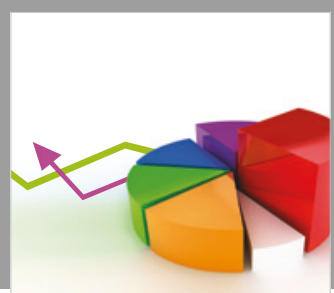

Journal of

Probability and Statistics
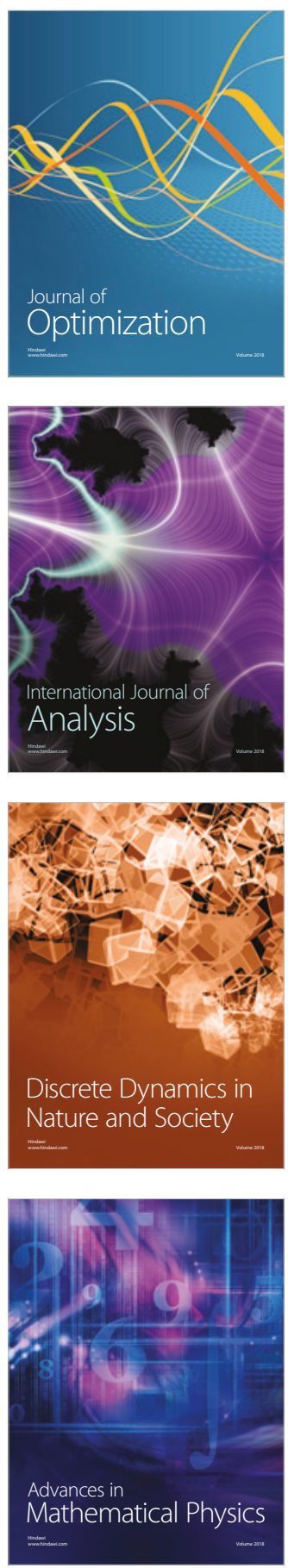